\title{
Editorial
}

\section{COVID-19: Cardio-pulmonary and Vascular Manifestations}

\author{
Meinrad Gawaz ${ }^{1}$ Rüdiger E. Scharf $2,3,4$ \\ ${ }^{1}$ Department of Cardiology and Angiology, Eberhard Karls University \\ Tübingen, Tübingen, Germany \\ 2 Program in Cellular and Molecular Medicine, Boston Children's \\ Hospital, Boston, MA, USA \\ 3 Department of Pediatrics, Harvard Medical School, Boston, MA, USA \\ ${ }^{4}$ Division of Experimental and Clinical Hemostasis, Hemotherapy, and \\ Transfusion Medicine, and Hemophilia Comprehensive Care Center, \\ Institute of Transplantation Diagnostics and Cell Therapy, Heinrich \\ Heine University Medical Center, Düsseldorf, Germany
}

Hamostaseologie 2021;41:347-348.

\section{Abstract}

The COVID-19 pandemic is still threatening us, our patients, and the global health care system. Since the first outbreak at the end of 2019 in China, it became rapidly clear that a new variant of a SARS virus, SARS-CoV-2, is threatening our human society worldwide. Since then, the scientific community has accumulated an incredibly large amount of knowledge about the pathophysiology of this virus, primarily affecting the respiratory tract and, in severe cases, subsequently resulting in acute respiratory distress syndrome and multiple organ failure due to uncontrolled systemic inflammatory response syndrome. ${ }^{1,2}$
It became evident shortly after the initial outbreak of COVID19 that not all individuals are equally affected. Patients with preexisting cardiovascular disease and elder individuals are at higher risk for a severe course of COVID-19. ${ }^{1}$ Furthermore, the SARS-CoV-2 infection may not exclusively induce respiratory failure but also compromise the cardiovascular system leading to myocardial infarction, myocarditis, organ failure, and severe thrombosis-associated diseases such as deep vein thrombosis and pulmonary embolism including thrombosis of the cerebral veins. ${ }^{1,3}$ It has been recognized that SARS$\mathrm{CoV}-2$ utilizes the angiotensin-converting enzyme 2 receptor for cell entry consecutively altering physiologic functions of this receptor and causing damage of the affected organs.

Coronaviruses are positive single-stranded, enveloped RNA viruses that can infect both humans and animals. SARS-CoV-2 belongs to the beta coronavirus subfamily. It is one of three members of this subfamily that are able to infect humans, causing severe respiratory, gastrointestinal, or central nervous infections. SARS-CoV-2 has close (79.6\%) genetic similarity to SARS-CoV, the coronavirus that caused the SARS pandemic in 2002/2003. The SARS-CoV-2 virus has around 50 to $200 \mathrm{~nm}$ in diameter. SARS-CoV-2 basically consists of four structural proteins including the $\mathrm{S}$ (spike), $\mathrm{E}$ (envelope), $\mathrm{M}$ (membrane), and $\mathrm{N}$ (nucleocapsid) proteins. The $\mathrm{N}$ protein integrates the RNA, and the other three proteins (S, E, M) built up the viral envelope. Moreover, the spike protein shows a 96.0\% genomic sequence identity with the SARS-related CoV RaTG13, a bat coronavirus. ${ }^{1}$ The spike protein mediates attachment to and entry into host cells. Besides that, the spike is the component of the virus that defines host and tissue tropism and causes antigenicity. Consequently, the spike protein is the target immunogen of most of currently available COVID-19 vaccines.

The present issue of Haemostaseologie-Progress in Haemostasis focuses on COVID-19 and cardiovascular diseases. Early cardiovascular risk assessment of SARS-CoV-2-positive patients is of utmost importance of patient care. The article by Zdanyte and Rath summarizes the latest reports on aspects of systemic inflammatory reaction following respiratory infection on elevation of pulmonary artery pressure and failure of the right ventricle. Although the pathogenesis is unclear, impaired left and right ventricular function in the early phase of COVID-19 is associated with poor clinical outcome. Thus, a consequent and guideline-oriented therapy considering the underlying cardiovascular dysfunction(s) is warranted in the treatment of COVID-19.4

Elevated cardiac biomarkers are common findings in patients with severe COVID-19 infection. ${ }^{1}$ The assessment of troponin may improve risk prediction and timely therapy in COVID-19. In our comprehensive review, Mizera et al present an overview of the incidence, potential mechanisms, and
Address for correspondence Meinrad Gawaz, Department of Cardiology and Angiology, Eberhard Karls Universität Tübingen, Tübingen, Germany (e-mail: Meinrad.Gawaz@med. uni-tuebingen.de). (c) 2021. Thieme. All rights reserved. Georg Thieme Verlag KG,

Rüdigerstraße 14,

70469 Stuttgart, Germany

Received: August 11, 2021

Accepted: August 11, 2021
DOI https://doi.org/ 10.1055/a-1370-0231. ISSN 0720-9355. 
outcome of acute cardiac injury in COVID-19. Acute cardiac injury is associated with coagulation abnormalities in sepsis and altered immune response. Further endothelial damage and inflammation ("endotheliitis") has been recognized as important feature of enhanced vascular dysfunction and thrombosis. ${ }^{5}$ Specifically, sustained fulminant endothelial cell activation during severe COVID-19 causes markedly increased von Willebrand factor (VWF) levels, ${ }^{6,7}$ and plasma VWF propeptide has been proposed as a biomarker in this setting. ${ }^{8,9}$

Patients with SARS-CoV-2 infection show an unusual wide spectrum of increased troponin levels. Severe and prolonged troponin elevation is observed in SARS-CoV2-infected humans in the absence of unstable coronary artery disease. ${ }^{1}$ Thus, a direct myocardial inflammation either caused by systemic hyperinflammation or a more directly virus-induced effect is discussed. Histopathological and imaging (cardio-MR) abnormalities have been well documented in COVID-19 patients, although the pathophysiology remains obscure. Greulich at al. discuss this important aspect in their article. ${ }^{10}$

Myocarditis may be present even in asymptomatic people, a finding that has been recognized by cardio-MR studies. Myocarditis is often associated with elevated troponin and affected individuals are at enhanced risk for sudden cardiac death and rhythm disturbances. Common cardiac arrhythmias in COVID-19 patients include sinus tachycardia, atrial fibrillation (AF), ventricular tachycardia, ventricular fibrillation, atrioventricular block, sinus nodal block, or QTc prolongation. AF is the most common heart rhythm disorder. The importance of mindfulness for rhythm disturbances in care of COVID-19 is highlighted by Duckheim et al. ${ }^{11}$

Enhanced platelet activity has been described to contribute to thromboinflammation in a variety of pathogen-related and "sterile" inflammatory diseases. In COVID-19 patients, platelet count differs between mild and serious infections. Patients with mild symptoms have a slightly increased platelet count, whereas thrombocytopenia is a hallmark of severe COVID-19 infections. Thrombocytopenia can be attributed to reduced platelet survival and severe thrombotic events. The most recent findings of platelets abnormalities in COVID-19 are summarized by Rohlfing et al. ${ }^{12}$

Hypercoagulability and vascular injury, which characterize morbidity in COVID-19, are frequently observed in the skin. The current review article by Gawaz et al. describes the clinical appearance of cutaneous vascular lesions and their association to COVID-19. The authors emphasize that clinicians need to be aware that skin manifestations may be the only and early symptom in SARS-CoV-2 infection. ${ }^{13}$

It is increasingly recognized that COVID-19 is associated with deep vein thrombosis, pulmonary embolism, and thrombosis of cerebral veins. Although it is not unexpected that severe viral infection triggers a prothrombotic disease state and development of clinical overt thrombosis, the recognition of thrombotic events in COVID-19-affected patients is increasing. In the present issue, two unusual case reports describe unexpected thrombotic events which enhance the awareness of this threatening disease following SARS-CoV-2 infection. Whether these case reports reflect just an association or a pathophysiological important aspect of SARS-CoV-2 infection remains, however, obscure. ${ }^{14,15}$
We trust that the articles of this theme issue contribute to improve the awareness of cardiovascular diseases in COVID-19 patients. Early assessment and consequent preventive (e.g., antithrombotic regimens) or therapeutic strategies (e.g., intensified heart failure treatment) and the awareness of threatening rhythm abnormalities are a critical cornerstone in successful patient care of COVID-19. We are thankful to the authors for their diligent contributions and also appreciate the substantial efforts of the reviewers and of Dr. Elinor Switzer, who, working on backstage, greatly helped improving the quality of submitted papers. Several other COVID-19 manuscripts scheduled for this edition are still in revision or in preparation and will be published in an upcoming issue of Hämostaseologie- Progress in Haemostasis.

\section{Conflict of interest}

The author declares that he has no conflict of interest.

\section{References}

1 Guzik TJ, Mohiddin SA, Dimarco A, et al. COVID-19 and the cardiovascular system: implications for risk assessment, diagnosis, and treatment options. Cardiovasc Res 2020;116(10):1666-1687

2 Mueller KAL, Langnau C, Günter M, et al. Numbers and phenotype of non-classical CD14 ${ }^{\mathrm{dim}} \mathrm{CD} 16^{+}$monocytes are predictors of adverse clinical outcome in patients with coronary artery disease and severe SARS-CoV-2 infection. Cardiovasc Res 2021;117(01):224-239

3 Harrison SL, Buckley BJR, Rivera-Caravaca JM, Zhang J, Lip GYH. Cardiovascular risk factors, cardiovascular disease, and COVID19: an umbrella review of systematic reviews. Eur Heart J Qual Care Clin Outcomes 2021;7(04):330-339

4 Zdanyte M, Rath D. Cardiovascular risk assessment in COVID-19. Hamostaseologie 2021;41:348-353

5 Mizera L, Borst O. COVID-19 and the incidence of acute myocardial injury. Hamostaseologie 2021;41:354-362

6 Escher R, Breakey N, Lammle B. ADAMTS13 activity, von Willebrand factor, factor VIII and D-dimers in COVID-19 inpatients. Thrombosis research 2020;192:174-175

7 Escher R, Breakey N, Lammle B. Severe COVID-19 infection associated with endothelial activation. Thrombosis research 2020;190:62

8 Mancini I, Baronciani L, Artoni A, et al. The ADAMTS13-von Willebrand factor axis in COVID-19 patients. Journal of thrombosis and haemostasis: JTH 2021;19:513-521

9 Ward SE, Curley GF, Lavin M, et al. Von Willebrand factor propeptide in severe coronavirus disease 2019 (COVID-19): evidence of acute and sustained endothelial cell activation. British journal of haematology 2021;192:714-719

10 Greulich S, Klingel K. COVID-19 and myocarditis: findings from cardiac magnetic resonance imaging and endomyocardial biopsies. Hamostaseologie 2021;41:364-368

11 Duckheim M, Schreieck J. COVID-19 and cardiac arrhythmias. Hamostaseologie 2021;41:369-375

12 Rohlfing A-K, Rath D, Geisler T, Gawaz M. Platelets and COVID-19. Hamostaseologie 2021;41:376-382

13 Gawaz A. Microvascular skin manifestations caused by COVID-19. Hamostaseologie 2021;41:383-392

14 Pravdic Z, Bogdanovic A, Virijevic M, Sabljic N, Pantic N, Vukovic NS. COVID-19 presented with deep vein thrombosis in a patient with paroxysmal nocturnal haemoglobinuria. Hamostaseologie 2021;41:394-396

15 Appenzeller FF, Schmehl J, Gawaz M, Müller II. Extensive thromboembolism in a young male with asymptomatic COVID-19 infection and heterozygous factor V Leiden mutation. Hamostaseologie 2021; 41:398-400 